BASIC SCIENCE ARTICLE

\title{
LED-phototherapy does not induce oxidative DNA damage in hyperbilirubinemic Gunn rats
}

Lori W. E. van der Schoor ${ }^{1}$, Christian V. Hulzebos ${ }^{2}$, Martijn H. van Faassen ${ }^{3}$, Ido P. Kema ${ }^{3}$, Alain de Bruin ${ }^{1,4}$, Rick Havinga ${ }^{1}$, Mirjam Koster ${ }^{1}$, Sameh A. Youssef ${ }^{4}$, Laura Bongiovanni ${ }^{4}$, Johan W. Jonker ${ }^{1}$ and Henkjan J. Verkade ${ }^{1}$

BACKGROUND: Phototherapy (PT) is the standard treatment of neonatal unconjugated hyperbilirubinemia. Fluorescent tube (FT)emitted PT light is known to induce oxidative DNA damage in neonates. Nowadays, however, FTs have largely been replaced by light-emitting diodes (LEDs) for delivering PT. Until now, it is unknown whether LED-PT causes oxidative DNA damage. We aim to determine whether LED-PT induces oxidative DNA damage in hyperbilirubinemic rats.

METHODS: Adult Gunn rats, with genetically unconjugated hyperbilirubinemia, received LED-PT in the clinically relevant doses of 10 or $30 \mu \mathrm{W} / \mathrm{cm}^{2} / \mathrm{nm}$. Urine was collected at 0,24 , and $48 \mathrm{~h}$ of PT. A group of young Gunn rats received intensive LED-PT of $100 \mu \mathrm{W} /$ $\mathrm{cm}^{2} / \mathrm{nm}$ for $24 \mathrm{~h}$. Urine was collected every $8 \mathrm{~h}$ and analyzed for the levels of oxidative DNA damage marker 8-hydroxy2 'deoxyguanosine (8-OHdG) and creatinine. DNA damage was evaluated by immunohistochemistry $(\mathrm{\gamma H} 2 \mathrm{AX})$ of skin and spleen samples.

RESULTS: LED-PT of 10 and $30 \mu \mathrm{W} / \mathrm{cm}^{2} / \mathrm{nm}$ did not affect urinary concentrations of 8-OHdG and creatinine or the 8-OHdG/ creatinine ratio. Likewise, intensive LED-PT did not affect the $8-\mathrm{OHdG} / \mathrm{creatinine}$ ratio or the number of $\mathrm{\gamma H} 2 \mathrm{AX}$-positive cells in the skin or spleen.

CONCLUSIONS: Our results show that LED-PT does not induce oxidative DNA damage in hyperbilirubinemic Gunn rats either at clinically relevant or intensive dosages.

Pediatric Research (2019) 85:1041-1047; https://doi.org/10.1038/s41390-019-0367-y

\section{INTRODUCTION}

Bilirubin is a potentially neurotoxic metabolite. Severe unconjugated hyperbilirubinemia is associated with the development of kernicterus, resulting in permanent neurological damage or even death. Unconjugated hyperbilirubinemia occurs primarily in premature infants and the majority of infants with a gestational age $<32$ weeks is treated with phototherapy (PT). ${ }^{1}$

PT, which has been the standard therapy for unconjugated hyperbilirubinemia for over 60 years, converts unconjugated bilirubin (UCB) to structural and configurational photo-isomers that can be more readily secreted by the liver into the bile, and to a lesser extent, by the kidneys into urine. ${ }^{2}$ PT is assumed to be relatively safe and its widespread use has contributed to the decreased need for exchange transfusions. ${ }^{3}$ Some studies, however, have suggested an increased mortality risk by PT, especially in sick (extremely) low birth weight (E)LBW infants. ${ }^{4}$ Furthermore, in recent years, associations have been reported between PT in neonatal life and diseases occurring later in life, such as diabetes, asthma, epilepsy, and even childhood cancer. ${ }^{5-7}$

Known cancer-inducing agents, such as tobacco and ultraviolet (UV) radiation, exert their toxic effects partially by induction of oxidative stress and oxidative DNA damage. When oxidative DNA damage exceeds the repair capacity, permanent DNA changes occur. Preterm infants are known to be predisposed to several oxidative stress-mediated diseases (e.g., bronchopulmonary dysplasia and retinopathy of prematurity). ${ }^{8}$ Preterm infants are particularly susceptible to oxidative damage due to significantly lower antioxidant concentrations at birth. ${ }^{9}$ Both the endogenous production and the materno-fetal placental transfer of antioxidants only occur in the last term of pregnancy. The ability of preterms to produce antioxidants in response to oxidant challenges remains deficient in the postnatal period. ${ }^{9}$ It has even been suggested that mild jaundice has a physiologic function through the protective effects of UCB, which is a proven antioxidant, at least in vitro and in blood. ${ }^{10}$ In this way, mildly jaundiced infants could be protected against oxidative stressors, such as, e.g., oxygen treatment ${ }^{8}$ or potentially PT. However, severe hyperbilirubinemia induces oxidative stress and bilirubin-induced neurotoxicity in the brain. ${ }^{11-14}$ These arguments underline the importance of studying these stressors in a hyperbilirubinemic environment.

PT has been associated with increased lipid peroxidation in (E) LBW neonates, which could be the result of increased oxidative stress. ${ }^{15}$ Several studies show induced DNA damage in white blood cells (WBCs) after PT. ${ }^{16,17}$ In these studies, PT light has been emitted by fluorescent tubes (FTs), whereas currently used PT devices are often equipped with light-emitting diodes (LEDs). LEDPT allows much higher intensity treatment with less heat

\footnotetext{
${ }^{1}$ Section of Molecular Metabolism and Nutrition, Department of Pediatrics, University of Groningen, University Medical Center Groningen, Groningen, The Netherlands; ${ }^{2}$ Division of Neonatology, Department of Pediatrics, University of Groningen, University Medical Center Groningen, Groningen, The Netherlands; ${ }^{3}$ Department of Laboratory Medicine,

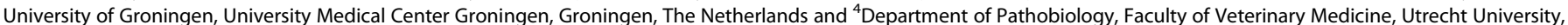
Utrecht, The Netherlands

Correspondence: Lori W. E. van der Schoor (l.w.e.van.der.schoor@umcg.nl)
}

Received: 10 December 2018 Revised: 18 February 2019 Accepted: 26 February 2019

Published online: 9 March 2019 
1042

production. Recently, intensive LED-PT with an irradiance intensity $>60 \mu \mathrm{W} / \mathrm{cm}^{2} / \mathrm{nm}$ has been applied for treating unconjugated hyperbilirubinemia in preterm infants and has proved to be highly effective. ${ }^{18}$ Until now, however, it has never been investigated whether LED-PT causes oxidative stress and DNA damage, especially when administered at these now available intensive dosages. This knowledge gap and concerns on the safety of highintensity LED-PT have impeded its clinical implementation. Here we aim to establish whether LED-PT induces oxidative DNA damage in hyperbilirubinemic Gunn rats at either conventional or at intensive treatment intensities.

\section{METHODS}

Animals

Homozygous adult Gunn rats (Gunn-UGT1A1 $1^{j} /$ BluHsdRrrc), which are genetically deficient of the bilirubin conjugation enzyme UDPglucuronosyltransferase 1A1, and wild-type (WT) littermates were obtained from the Rat Resource \& Research Center (Columbia, MO) and bred at the University Medical Center Groningen breeding colony (Groningen, The Netherlands). During all experiments, rats were allowed ad libitum access to water and RM3 diet (Special Diets Services, Essex, UK). Animals were kept in an environmentally controlled facility with a 12-h day/night cycle. All experiments were approved by the Animal Ethics Committee of the University Medical Center Groningen.

\section{Study design}

Conventional clinical PT intensity experiment. Gunn rats and WT rats ( $n=10$ and $n=8$ per group, respectively, 20-25 weeks of age) were individually placed in a urine collection cage for $30 \mathrm{~min}$. After the collection of minimal $200 \mu \mathrm{l}$ urine, animals were anesthetized using isoflurane, shaved on their back and flanks, and $100 \mu \mathrm{l}$ blood was collected from tail vein cut. Subsequently, the rats were group-housed in a conventional cage placed under a mavi LED ${ }^{\circledR}$ Phototherapy System (Inspiration Healthcare, Crawley, UK) (wavelength range $450-520 \mathrm{~nm}$, peak $450-470 \mathrm{~nm}$ ) for $24 \mathrm{~h}$. Intensities were measured at the bottom of the cage and set on 10 or $30 \mu \mathrm{W} / \mathrm{cm}^{2} / \mathrm{nm}$, respectively. After $24 \mathrm{~h}$ of PT, new blood and urine samples were collected as described above.

Intensive PT experiment. Gunn rats ( $n=6$ per group, 8 weeks of age) were individually placed on a grid in a metabolic cage for 24 $\mathrm{h}$, with water and chow ad libitum. After a $24 \mathrm{~h}$ acclimatization period, the rats were anesthetized using isoflurane, shaved on their back and flanks, and $100 \mu \mathrm{l}$ blood was collected from the tail vein. Depending on the group, animals were put under a LED-PT device of $100 \mu \mathrm{W} / \mathrm{cm}^{2} / \mathrm{nm}$ (kindly provided by Professor Henk Vreman, Stanford University, Stanford, CA; $100 \mu \mathrm{W}$ group) (wavelength range $416-544 \mathrm{~nm}$, peak $467 \mathrm{~nm}$ ) or left anesthetized for $20 \mathrm{~min}$ (Control group). As a positive control, these young Gunn rats were radiated with $3.5 \mathrm{~Gy}$ of $y$-radiation. This sublethal dose is known to induce both 8-OHdG in urine and $\mathrm{yH} 2 \mathrm{AX}$ in mice. ${ }^{19,20}$ After anesthesia, the rats were placed in the urine collection cage for another $24 \mathrm{~h}$. The $100 \mu \mathrm{W}$ group was placed in the urine collection cage with a PT lamp placed on top, intensity was measured to be $100 \mu \mathrm{W}$ in the entire cage, using a Biliblanket Lightmeter II (GE Healthcare, Madison, WI). Urine samples were collected every $8 \mathrm{~h}$. After $24 \mathrm{~h}$, animals were anesthetized using isoflurane. Blood was collected from the tail vein and the liver, spleen and skin were collected and stored in $4 \%$ formaldehyde for $24 \mathrm{~h}$ before processing. Subsequently, rats were terminated by decapitation under anesthesia.

\section{Urinary 8-hydroxy-2'deoxyguanosine (8-OHdG)}

$8-\mathrm{OHdG}$ is the breakdown product of guanine, the most oxidativestress-susceptible base in DNA. 8-OHdG appears only after oxidative DNA damage and is quantitatively excreted into urine.
Consequently, 8-OHdG is not only a non-invasive marker for oxidative stress but is also specific for oxidative-stress-induced DNA damage. ${ }^{21}$ Two hundred $\mu \mathrm{l}$ of urine was transferred to a $96-$ well plate and $300 \mu \mathrm{l}$ internal standard $\left(25 \mathrm{ng} / \mathrm{ml} 8-O H d G-{ }^{15} \mathrm{~N}_{5}\right.$ in $50 \mathrm{mM} \mathrm{NH}_{4} \mathrm{Ac}, \mathrm{pH}$ 4.7) was added to each well. After vortexing for 1 min, samples were transferred to an Oasis HLB SPE column (Waters, Millford, MA), preconditioned with $1 \mathrm{ml} \mathrm{MeOH}$ and $1 \mathrm{ml}$ $50 \mathrm{mM}$ NH4Ac, pH 4.7 buffer, and vacuum was applied using $\mathrm{N}_{2}$ gas. The column was washed with $500 \mu \mathrm{l}$ buffer and the sample was eluted in $500 \mu \mathrm{l} \mathrm{MeOH}$. Subsequently, the eluate was evaporated under $\mathrm{N}_{2}$ gas at $50^{\circ} \mathrm{C}$ for $15 \mathrm{~min}$ and redissolved in $200 \mu \mathrm{l} 80 \%$ acetonitrile. $8-\mathrm{OHdG}$ was measured by liquid chromatography and isotope dilution tandem mass spectrometry (LC-MS/MS). Ten $\mu \mathrm{l}$ of the sample solution was injected into the LC-MS/MS instrument. Chromatography was performed using a XBridge Amide column $(50 \times 3.0 \mathrm{~mm}, 2.5 \mu \mathrm{m}$, Waters $)$ and mass spectrometric detection by a XEVO TQ-MS (Waters).

\section{Urinary creatinine analysis}

Urinary creatinine was enzymatically determined by the general diagnostics laboratory in the UMCG, using a Roch/Hitachi Cobas C 501 analyzer (Hoffman-La Roche, Basel, Switzerland/Hitachi, Tokyo, Japan). For each urine sample, the $8-\mathrm{OHdG}$ concentration was divided by the creatinine concentration to obtain the $8-\mathrm{OHdG} /$ creatinine ratio. Thereby, the $8-\mathrm{OHdG}$ concentration was corrected for urine osmolality. For clarity, the $8-\mathrm{OHdG}$ concentration is displayed in $\mathrm{ng} / \mathrm{ml}$ and the creatinine concentration in $\mathrm{mmol} / \mathrm{l}$. The ratio is displayed in $\mu \mathrm{g} / \mathrm{g}$ creatinine, which is calculated by dividing the $8-\mathrm{OHdG}$ concentration in $\mathrm{ng} / \mathrm{ml}$ by the creatinine concentration in $\mathrm{g} / \mathrm{l}$ (concentration in $\mathrm{mmol} / \mathrm{l}$ multiplied by 0.11312 )

\section{Gamma-H2AX}

$\gamma \mathrm{H} 2 \mathrm{AX}$ is a phosphorylated histone protein, which appears at the site of double-strand DNA breaks (DSBs) as soon as DNA repair is initiated. $\mathrm{\gamma H} 2 \mathrm{AX}$ is a widely used DNA damage marker in tissues for research purposes and is more sensitive than conventional assays (e.g., Comet assay, neutral elution or two-dimensional gel electrophoresis). ${ }^{22}$ Four- $\mu$ m paraffin-embedded tissue sections were dried for $1 \mathrm{~h}$ at $55^{\circ} \mathrm{C}$. Sections were deparaffinized in xylene and rehydrated in a graded series of alcohol. The antigen retrieval was performed using $1 \mathrm{mM}$ EDTA/10 mM Tris-HCL buffer, $\mathrm{pH}$ 9, in a microwave and sections were left to cool to room temperature. Endogenous peroxidase activity was blocked with $1 \% \mathrm{H}_{2} \mathrm{O}_{2}$ in methanol for $30 \mathrm{~min}$. The unspecific antigens on tissues were blocked in $10 \%$ normal goat serum for $30 \mathrm{~min}$. Then the sections were incubated with primary antibody (rabbit anti H2AX (s139) (20E3), Cell Signaling Technology, Danvers, MA) at $4{ }^{\circ} \mathrm{C}$ overnight and incubated with biotinylated goat-anti-rabbit secondary antibody (biotinylated Goat Anti-Rabbit IgG Antibody, BA-1000, Vector Laboratories, Peterborough, UK) at 1:250 for $30 \mathrm{~min}$ at room temperature. After 30-min incubation with Avidin-biotin $A B C$ complex (PK-4000, Vector), the sections were washed and colorized with 3,3'-diaminobenzidine (Sigma, D5637) for $10 \mathrm{~min}$ and counterstained with hematoxylin. Positive cells were manually counted using ImageJ Cell Counter Plugin (National Institute of Health, Bethesda, MD) analyzing pictures of 10 randomly selected high- power fields $(\times 40)$ for each sample. We analyzed $\mathrm{yH} 2 \mathrm{AX}$ in a superficial tissue, skin, and an internal tissue, spleen. We chose spleen because it is the main site of WBC sequestration and previous studies have shown that FT-PT causes DNA damage in WBCs.

\section{Bilirubin}

Blood was collected in EDTA-coated capillaries and kept in EDTAcoated tubes (MiniCollect, Greiner Bio-One, Kremsmünster, Austria) in the dark till centrifugation. Plasma was stored under argon in amber-colored containers at $-80^{\circ} \mathrm{C}$, till analysis. Bilirubin 

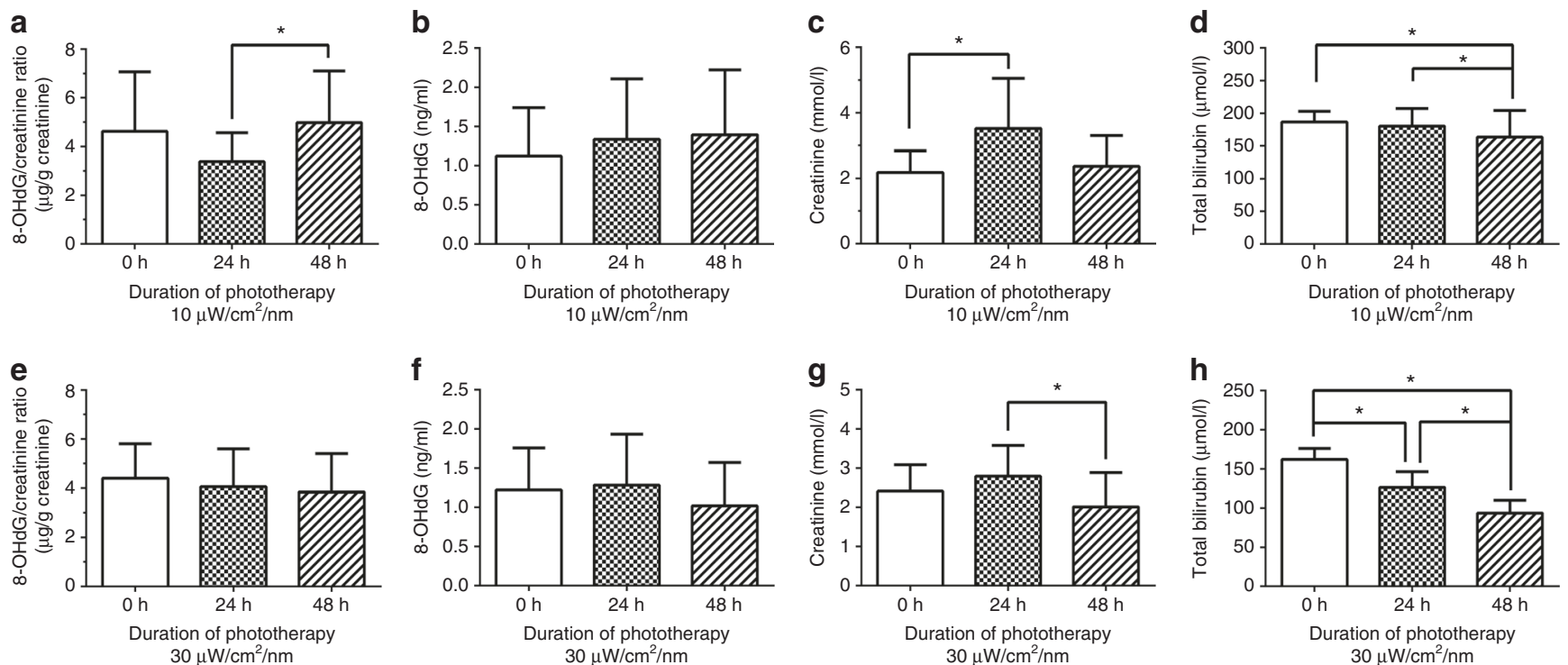

Fig. 1 Conventional phototherapy (PT) intensities do not induce the 8-hydroxy-2'deoxyguanosine (8-OHdG)/creatinine ratio. a The 8-OHdG/ creatinine ratio before and after 24 and $48 \mathrm{~h}$ low-intensity PT of $10 \mu \mathrm{W} / \mathrm{cm}^{2} / \mathrm{nm}$. b The urinary $8-O H d G$ concentration before and after 24 and $48 \mathrm{~h}$ low-intensity PT. c The urinary creatinine concentration before and after 24 and $48 \mathrm{~h}$ low-intensity PT. d Total bilirubin (TB) before and after 24 and $48 \mathrm{~h}$ low-intensity PT. e The $8-\mathrm{OHdG} / \mathrm{creatinine}$ ratio before and after 24 and $48 \mathrm{~h}$ high-intensity PT of $30 \mu W / \mathrm{cm}^{2} / \mathrm{nm}$. f The urinary $8-\mathrm{OHdG}$ concentration before and after 24 and $48 \mathrm{~h}$ high-intensity PT. $\mathrm{g}$ The urinary creatinine concentration before and after 24 and $48 \mathrm{~h}$ high intensity PT. $\mathbf{h}$ TB before and after 24 and $48 \mathrm{~h}$ high-intensity PT. (error bars represent standard deviation (SD)) ${ }^{*} p<0.05$

was quantified using the colorimetric diazo method, using a Roch/ Hitachi Cobas C 501 analyzer.

\section{Statistics}

Paired comparisons were tested for significance using Wilcoxon rank test. Unpaired comparisons were statistically tested using Mann-Whitney $U$ analysis. Significance was considered reached at $p<0.05$. Statistical tests were performed using GraphPad Prism 6.0 (GraphPad Software, La Jolla, CA).

\section{RESULTS}

LED-PT does not increase markers of oxidative DNA damage at conventional intensities

To assess the effect of LED-PT on oxidative DNA damage, we treated Gunn rats with 2 commonly used PT doses 10 (low intensity) and $30 \mu \mathrm{W} / \mathrm{cm}^{2} / \mathrm{nm}$ (high intensity), respectively. Figure 1 shows that neither low (Fig. 1a-c) nor high (Fig. 1e-g) intensity PT increased the levels of $8-\mathrm{OHdG}$ and creatinine or the $8-\mathrm{OHdG} /$ creatinine ratio. As expected, PT did significantly decrease the levels of plasma total bilirubin (TB) after $48 \mathrm{~h}$ by $12 \%$ and by $22 \%$ at low and high intensities, respectively (Fig. 1d-h).

Intensive PT does not induce oxidative DNA damage Intensive PT with intensities up to $100 \mu \mathrm{W} / \mathrm{cm}^{2} / \mathrm{nm}$ are increasingly being applied in research settings not only in neonates but also in patients with Crigler-Najjar type $1 .^{18}$ To assess its potential toxicity, we studied the effects of intensive LED-PT of $100 \mu \mathrm{W} / \mathrm{cm}^{2} /$ $\mathrm{nm}$. Intensive PT did not affect the $8-\mathrm{OHdG} / \mathrm{creatinine}$ ratio at $24 \mathrm{~h}$ (Fig. 2a) or at any earlier time point (Fig. S1). Interestingly, we found significantly lower $8-\mathrm{OHdG} /$ creatinine levels in PT-treated rats as compared to control rats, but the $8-\mathrm{OHdG} / \mathrm{creatinine}$ ratios in PT rats were not significantly decreased when compared to their starting levels. The absence of any PT effect on these internal controls makes this a probable coincidental finding. We did observe a significant induction of both urinary $8-\mathrm{OHdG}$ and creatinine (Fig. 2b, c), indicating that urine becomes more concentrated upon PT treatment. This increase was not observed in the control group. In order to explain the increased urine creatinine concentration in the PT group, we monitored water intake, food intake, urine volume, and body weight. PT significantly decreased food and water intake, resulting in a significantly lower urine production (Fig. S2), leading to more concentrated urine with a higher creatinine concentration. Intensive PT strongly decreased TB concentration (by $85 \%$ at 24 h) to a mean plasma concentration of $5.8 \mu \mathrm{mol} / \mathrm{l}$, which is within the physiological range of adult humans.

In addition to urinary analyses, we determined a parameter of DNA damage in tissue. Figure $2 \mathrm{e}$ shows the number of $\mathrm{pH} 2 \mathrm{AX}$-positive cells in the skin of intensive PT-treated and control rats. Unexpectedly, we observed a significant decrease of $\mathrm{\gamma H} 2 \mathrm{AX}$-positive cells in PT-treated rats. Most $\mathrm{\gamma H} 2 \mathrm{AX}$-positive cells were found in the follicular epithelium. In the spleen, PT did not affect the number of $\mathrm{\gamma H} 2 \mathrm{AX}$-positive cells (Fig. 2f). As a positive control, we show that $3.5 \mathrm{~Gy} \gamma$ radiation caused a significant 86 -fold increase in $\mathrm{\gamma H} 2 \mathrm{AX}$ foci, as well as a significant increase in the $8-\mathrm{OHdG} /$ creatinine ratio (Fig. S3).

Serum bilirubin concentration does not correlate with the urinary 8-OHdG/creatinine ratio

To assess a potential anti-oxidative effect of bilirubin, we compared the basal $8-\mathrm{OHdG} / \mathrm{creatinine}$ ratio between Gunn and WT rats. We observed no difference in the ratio and $8-\mathrm{OHdG}$ or creatinine concentration (Fig. 3). When comparing the basal bilirubin levels of the old and the young Gunn rats, however, we observed a significantly higher $8-\mathrm{OHdG} / \mathrm{creatinine}$ ratio in the latter (Fig. 3d). Upon closer examination of the separate ratio constituents, both the $8-\mathrm{OHdG}$ and creatinine concentration were increased in young Gunn rats (Fig. 3e, f). Importantly, we observed no correlation between the ratio and TB at low intensity, high intensity, or intensive PT (Fig. 3h-j).

\section{DISCUSSION}

We are the first to demonstrate that LED-PT up to $100 \mu \mathrm{W} / \mathrm{cm}^{2} / \mathrm{nm}$, an intensity that exceeds conventional FT-PT, does not induce oxidative DNA damage in hyperbilirubinemic Gunn rats. 


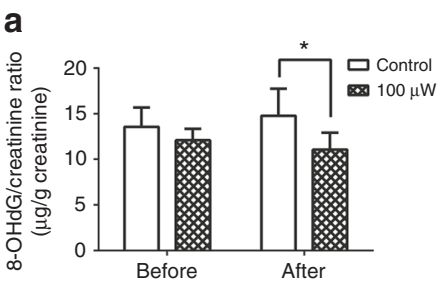

b

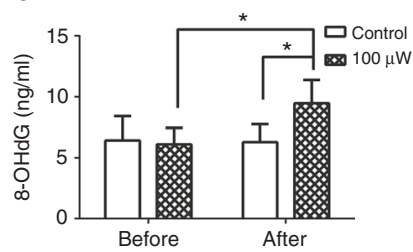

C

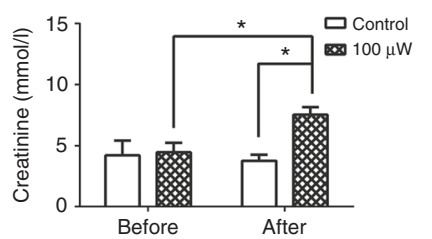

d

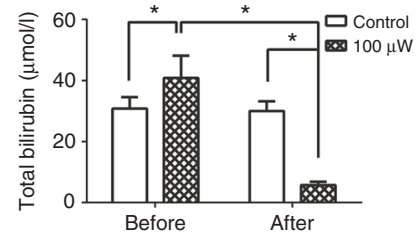

$\mathbf{e}$
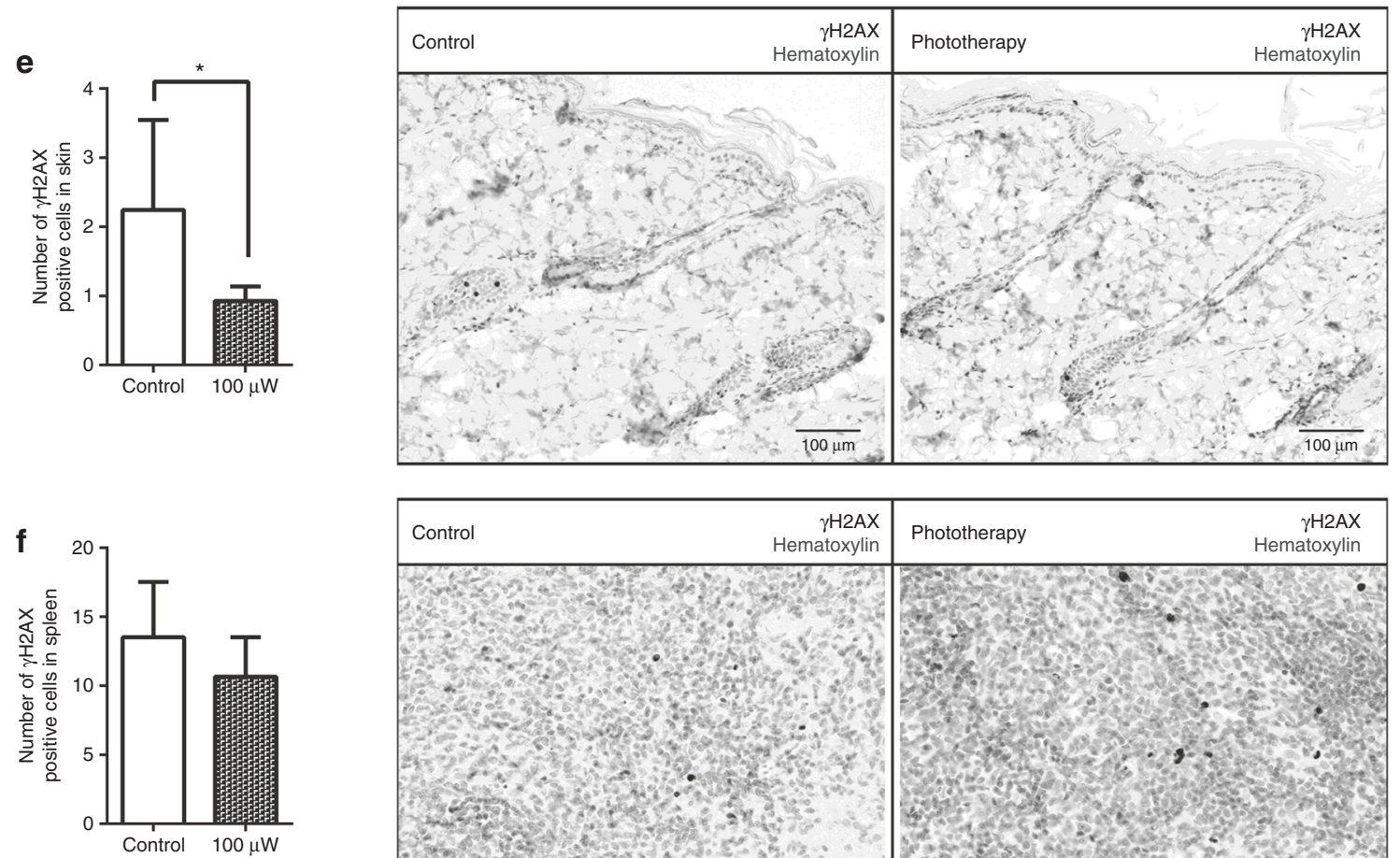

Fig. 2 Intensive phototherapy (PT) does not induce the 8-hydroxy-2'deoxyguanosine (8-OHdG)/creatinine ratio or $\gamma \mathrm{H} 2 \mathrm{AX}$ in tissue. a The 8$\mathrm{OHdG} /$ creatinine ratio before and after $24 \mathrm{~h}$ intensive PT of $100 \mu \mathrm{W} / \mathrm{cm}^{2} / \mathrm{nm}$ or control interventions. $\mathbf{b}$ The urinary 8 -OHdG concentration before and after $24 \mathrm{~h}$ intensive PT or control interventions. c The urinary creatinine concentration before and after $24 \mathrm{~h}$ intensive PT or control interventions. d Total bilirubin before and after $24 \mathrm{~h}$ intensive PT or control interventions. e The number of $\gamma \mathrm{H} 2 \mathrm{AX}$-positive cells in the skin after $24 \mathrm{~h}$ intensive PT or control interventions (average number of cells in 10 manually counted high-power fields (HPFs)). $\mathbf{f}$ The number of $\gamma \mathrm{H} 2 \mathrm{AX}$-positive foci in the spleen after $24 \mathrm{~h}$ intensive PT or control interventions (average number of cells in 10 manually counted HPFs). (error bars represent SD) ${ }^{*} p<0.05$

We used two markers that are directly correlated with DNA damage. 8-OHdG is the direct oxidation product of the DNA base guanine, the most susceptible DNA base, and is therefore directly correlated with DNA oxidation. 8-OHdG is a non-invasive marker, which allows not only serial measurements over time but will also allow us to extend our study to a clinical setting with preterm neonates, in which the options of obtaining invasive samples (blood, tissues) are limited.

Because the $8-\mathrm{OHdG} / \mathrm{creatinine}$ ratio does not show actual DSBs, we added $\gamma \mathrm{H} 2 \mathrm{AX}$ as a second marker. $\mathrm{\gamma H} 2 \mathrm{AX}$ is a histone protein that is phosphorylated upon activation of the repair mechanism of DSBs. The number of $\gamma \mathrm{H} 2 \mathrm{AX}$-positive cells therefore directly correlates with DNA damage in tissues. ${ }^{22}$ Unexpectedly, $\mathrm{\gamma H} 2 \mathrm{AX}$ was not increased by PT in the skin, the most exposed tissue to PT. Instead, PT significantly decreased the number of $\gamma \mathrm{H} 2 \mathrm{AX}$-positive cells. Although we have no explanation for this unexpected decrease, there could be a direct effect of LED light on the hair follicle, as most $\mathrm{y}-\mathrm{H} 2 \mathrm{AX}$-positive cells are found in the follicular epithelium. LED light is known to promote hair regrowth and to increase hair tensile strength, which is hypothesized to be caused by vasodilation and improved follicular blood supply. ${ }^{23}$
Possibly, these changes could be accompanied by decreased $\gamma \mathrm{H} 2 \mathrm{AX}$ expression. The number of positive cells in the skin, however, was very small in both PT-treated and control animals, making the physiological relevance of this effect questionable.

We also analyzed DNA damage in the spleen, because it is an important organ in WBC sequestration and previous studies have shown DNA damage in WBCs. We, however, did not find an effect of PT on $\mathrm{YH} 2 \mathrm{AX}$. We hypothesize that this difference can be explained by our use of LED-PT versus conventional FT-PT, which has been used in virtually all currently available studies on this topic. Although FT devices have been commonly used in the past, clinically they are gradually being replaced by LED devices. LED-PT has been proven to be as effective as or superior to FT-PT. ${ }^{24}$ Our present data indicate that the high efficacy of LED-PT does not concur with indications of (more) oxidative stress. There are several reasons why FT-PT can be expected to cause more oxidative stress and DNA damage than LED-PT. First, the heat produced by FTs can induce hyperthermia, which is known to enhance oxidative stress. ${ }^{25}$ Second, FTs primarily produce UV light, which is subsequently converted to visible light by the internal phosphor coating. Several studies have described leakage of UV 

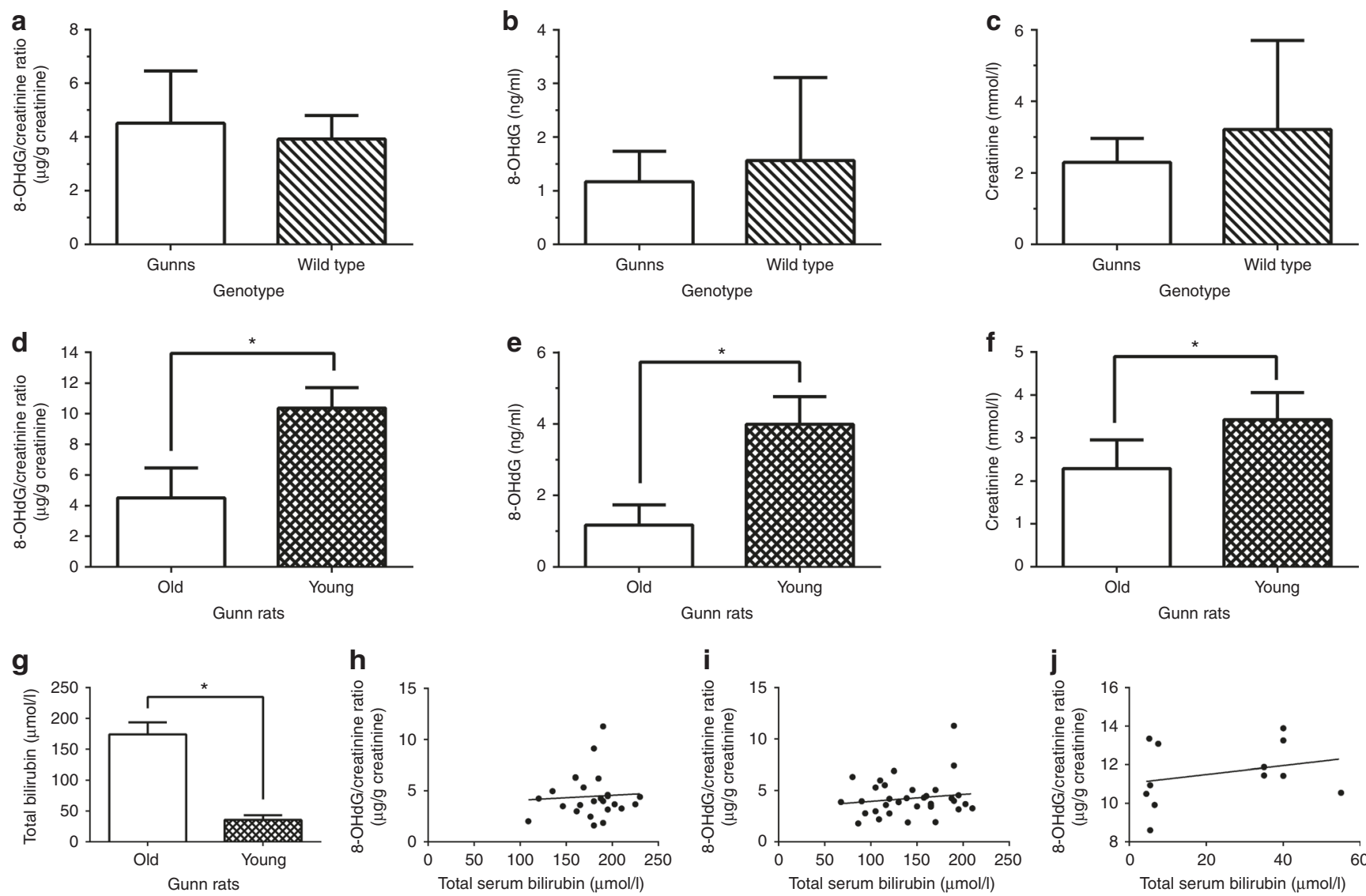

Fig. 3 The urinary 8-hydroxy-2'deoxyguanosine (8-OHdG)/creatinine ratio is not affected by bilirubin but decreases with age. a The 8-OHdG/ creatinine ratio in Gunn rats versus wild-type (WT) rats. b The urinary 8-OHdG concentration in Gunn rats versus WT rats. c The urinary creatinine concentration in Gunn rats versus WT rats. d The $8-\mathrm{OHdG} /$ creatinine ratio in old (20-25 weeks) versus young (8 weeks) Gunn rats. e The urinary 8-OHdG concentration in old versus young Gunn rats. $f$ The urinary creatinine concentration in old versus young Gunn rats. $\mathbf{g}$ The mean total bilirubin (TB) level in old versus young Gunn rats. $\mathbf{h}$ Correlation between the 8-OHdG/creatinine ratio and TB in Gunn rats treated with $10 \mu \mathrm{W} / \mathrm{cm}^{2} / \mathrm{nm}\left(r^{2}=0.004, p=0.754\right)$. $\mathbf{i}$ Correlation between the 8-OHdG/creatinine ratio and TB in Gunn rats treated with

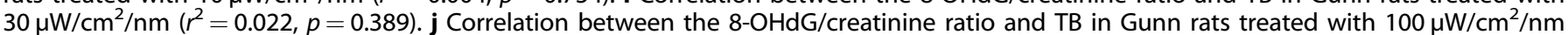
$\left(r^{2}=0.074, p=0.392\right)$. (error bars represent SD) ${ }^{*} p<0.05$

light from FTs, especially when the coating wears off and becomes damaged over time. ${ }^{26,27}$ This could theoretically lead to a clinically significant UV exposure, especially when the newborn infant is placed close to the lamp. Compared to LEDs, FTs need to be placed closer, due to the relatively lower irradiance of the emitted light, which could aggravate the exposure to UV. ${ }^{28}$ To protect from UV exposure, FT-based PT devices need to be used with a protective screen to absorb UV radiation. However, most studies do not report on the use of such a screen, and in practice, the screens may be removed after a certain time because they get dirty or damaged or because the protective role of the screens is not realized. Our study was performed with unfiltered LED light. Finally, FTs emit a broader wavelength of light compared to LEDs, and FTs emit several high irradiance peaks at different wavelengths. Although these are not visible by eye, these different wavelength peaks can potentially cause side effects, including the previously reported induction of oxidative stress or DNA damage. The blue LED spectrum, on the other hand, is narrower and does not have high irradiance peaks at wavelengths other than blue. ${ }^{29}$

There are two conflicting studies comparing conventional FT and LED-PT with regards to oxidative stress in term neonates. Demirel et al. describe an enhanced oxidative stress index after FTPT but not after LED-PT. In contrast, Kale et al. describes increased oxidative stress after both FT and LED-PT. ${ }^{30,31}$ In both studies, different treatment intensities are used for FT-PT (12-16 and $10-15 \mu \mathrm{W} / \mathrm{cm}^{2} / \mathrm{nm}$ by Demirel and Kale, respectively) and LED-PT
(30 and $60-90 \mu \mathrm{W} / \mathrm{cm}^{2} / \mathrm{nm}$ by Demirel and Kale, respectively), making any comparison difficult. Furthermore, both assess oxidative stress by measuring the total oxidant status (TOS) and total antioxidant capacity (TAC) in plasma by two colorimetric assays designed by Erel et al. From these two variables, the TOS: TAC ratio was calculated, representing the oxidative stress index. However, the validity of these assays can be questioned for this specific research question, since the methodological paper of the TOS assay describes interference of bilirubin with the assay. ${ }^{32}$ Moreover, since bilirubin is known to act as an antioxidant in blood, this could potentially affect TAC. PT could therefore hypothetically affect the oxidative stress index merely by decreasing plasma bilirubin. In our study, we circumvented these interactions by determining the physiologically relevant effect of oxidative stress, i.e., oxidative DNA damage, using markers that are only produced upon DNA damage. One other study reports on the effect of different colors of PT on 8-OHdG/creatinine in Gunn rats and concludes that blue PT causes a two-fold induction, whereas all other colors did not cause any significant change. However, since the induction is only shown in two rats without optimal controls, this methodology is questionable. ${ }^{33}$

To study the effect of hyperbilirubinemia on $8-\mathrm{OHdG}$ production in our model, we compared the basal $8-\mathrm{OHdG} / \mathrm{creatinine}$ ratio between age-matched Gunn rats and WT rats and we found no difference in the ratio or urinary $8-\mathrm{OHdG}$ and creatinine concentrations. Some studies have reported that bilirubin has 
antioxidant properties in vivo, which are hypothesized to be responsible for the inverse association between bilirubin and cardiovascular risk. However, the causality of these associations has been disputed, because the data have largely been obtained from either normobilirubinemic patients or from patients with Gilbert syndrome. ${ }^{34}$ The latter not only have mildly elevated bilirubin levels but also tend to be slimmer, with a metabolically more favorable phenotype, which could by itself decrease oxidative stress. ${ }^{35}$ High bilirubin levels, however, have been suggested to be toxic for cells and could thereby induce oxidative stress, at least when exceeding a cytotoxic threshold. ${ }^{36,37}$ This is especially true for brain, where bilirubin can cause profound neurotoxicity. ${ }^{11-14}$ Nevertheless, in our model bilirubin did not affect the urinary $8-\mathrm{OHdG} /$ creatinine ratio, indicating Gunn rats do not display altered sensitivity to oxidative stress.

Interestingly, when comparing old and young Gunn rats, we observed a significantly higher $8-\mathrm{OHdG} / \mathrm{creatinine}$ ratio in young Gunn rats. This seems counterintuitive, since oxidative damage is known to increase with age. ${ }^{38}$ One should be cautious, however, to compare Gunn rats of different ages for two reasons. First, creatinine is produced upon the breakdown of muscle cells and is therefore known to depend on muscle mass and age. As the muscle mass of rats changes with age, creatinine production also alters and so does the $8-\mathrm{OHdG} / \mathrm{creatinine}$ ratio. In addition to being a marker of urine osmolality, creatinine is also a marker of kidney function. The excretion and urinary levels of creatinine decrease when kidney function decreases. Gunn rats are known to suffer from bilirubin nephropathy, a progressively declining kidney function caused by the accumulation of bilirubin deposits in the kidney tissue. ${ }^{39}$ Therefore, their kidney function is expected to decline with age, which could affect the excretion of creatinine and possibly $8-\mathrm{OHdG}$.

\section{Limitations}

A limitation of this study is the fact that our urinary marker depends on kidney function. However, we corrected for kidney function by expressing DNA damage as the ratio of 8-OHdG over urinary creatinine. As the oxidative DNA damage marker, we chose to include $\mathrm{y}-\mathrm{H} 2 \mathrm{AX}$, but we did not include other oxidative stress or DNA damage markers, e.g., products of lipid or protein oxidation. Furthermore, our work does not contain a direct comparison between FT- and LED-PT. Also, we did not include $\gamma$-radiation or PT treatment for WT rats, since their basal 8-OHdG/creatinine was not altered compared to Gunn rats. In addition, although neonatal hyperbilirubinemia primarily occurs in preterm infants, we chose to perform this study in adult rats, instead of neonates. During the first postnatal week, when neonatal rats are still hairless, they depend on the warmth of their littermates and mother for their body heat and require almost continuous feeding. Administering accurate, reproducible, continuous PT would require separating the litter from each other and their mother, inducing stress, hypothermia, and food deprivation, all of which could induce oxidative stress. Lastly, owing to animal welfare considerations, this work only studied the effects of PT after the first 24-48 h of treatment. Although we do not observe any effects during this initial period, we cannot exclude potential side effects after longer PT exposure.

To our knowledge, this is the first study that dose-dependently determined the effects of LED-PT on oxidative DNA damage, including effects of intensive PT intensities up to $100 \mu \mathrm{W} / \mathrm{cm}^{2} / \mathrm{nm}$. These intensities are gaining clinical relevance, since more and more trials are studying the efficacy of intensive PT. ${ }^{18}$ Higherintensity PT leads to a significantly faster and larger bilirubin decrease than conventional treatment intensities. Shortening the duration of PT and enhancing the speed of bilirubin removal may have several theoretical advantages, including shorter exposure of the infant to toxic bilirubin levels, potentially less need for exchange transfusion, shorter hospitalization, and less interference in the mother-child interaction. Clinical implementation, however, has so far been impeded by concerns about DNA damage and previously reported long-term effects, including a tendency toward increased mortality in (E)LBW. This has led to studies analyzing the effects of intermittent PT and studies that investigate the effects of additional therapies next to $\mathrm{PT}^{40}$ Notwithstanding this important research, our work indicates that LED-PT does not cause oxidative DNA damage in hyperbilirubinemic rats and thereby may mitigate the previously raised concerns regarding that particular matter. Our study, however, does not exclude potential other harmful effects that could affect previously reported long-term adverse effects. Finally, it remains to be established whether our findings apply to preterm infants.

\section{ACKNOWLEDGEMENTS}

We thank Professor Henk Vreman for kindly providing the intensive LED phototherapy device.

\section{ADDITIONAL INFORMATION}

The online version of this article (https://doi.org/10.1038/s41390-019-0367-y) contains supplementary material, which is available to authorized users.

Competing interests: The authors declare no competing interests.

Publisher's note: Springer Nature remains neutral with regard to jurisdictional claims in published maps and institutional affiliations.

\section{REFERENCES}

1. Dennery, P. A., Seidman, D. S. \& Stevenson, D. K. Neonatal hyperbilirubinemia. N. Engl. J. Med. 344, 581-590 (2001).

2. Maisels, M. J. \& McDonagh, A. F. Phototherapy for neonatal jaundice. N. Engl. J. Med. 358, 920-928 (2008).

3. Brown, A. K., Kim, M. H., Wu, P. Y. \& Bryla, D. A. Efficacy of phototherapy in prevention and management of neonatal hyperbilirubinemia. Pediatrics 75, 393-400 (1985)

4. Arnold, C., Pedroza, C. \& Tyson, J. E. Phototherapy in ELBW newborns: does it work? Is it safe? Evid. Random. Clin. Trials 38, 452-464 (2014).

5. Dahlquist, G. \& Kallen, B. Indications that phototherapy is a risk factor for insulindependent diabetes. Diabetes Care 26, 247-248 (2003).

6. Maimburg, R. D., Olsen, J. \& Sun, Y. Neonatal hyperbilirubinemia and the risk of febrile seizures and childhood epilepsy. Epilepsy Res. 124, 67-72 (2016).

7. Wickremasinghe, A. C., Kuzniewicz, M. W., Grimes, B. A., McCulloch, C. E. \& Newman, T. B. Neonatal phototherapy and infantile cancer. Pediatrics https://doi. org/10.1542/peds.2015-1353 (2016).

8. Torres-Cuevas, I., Parra-Llorca, A. \& Sánchez-Illana, A. Oxygen and oxidative stress in the perinatal period. Redox Biol. 12, 674-681 (2017).

9. Davis, J. M. \& Auten, R. L. Maturation of the antioxidant system and the effects on preterm birth. Semin. Fetal Neonatal Med. 15, 191-195 (2010).

10. Stocker, R. Antioxidant activities of bile pigments. Antioxid. Redox Signal $\mathbf{6}$, 841-849 (2004).

11. Fujiwara, R., Nguyen, N., Chen, S. \& Tukey, R. H. Developmental hyperbilirubinemia and CNS toxicity in mice humanized with the UDP glucuronosyltransferase 1 (UGT1) locus. Proc. Natl. Acad. Sci. USA 107, 5024-5029 (2010).

12. Bortolussi, G. et al. Age-dependent pattern of cerebellar susceptibility to bilirubin neurotoxicity in vivo in mice. Dis. Model Mech. 7, 1057-1068 (2014).

13. Barateiro, A. et al. Reduced myelination and increased glia reactivity resulting from severe neonatal hyperbilirubinemia. Mol. Pharmacol. 89, 84-93 (2016).

14. Rawat, V., Bortolussi, G., Gazzin, S., Tiribelli, C. \& Muro, A. F. Bilirubin-induced oxidative stress leads to DNA damage in the cerebellum of hyperbilirubinemic neonatal mice and activates DNA double-strand break repair pathways in human cells. Oxid. Med. Cell. Longev. 1801243, 11 (2018).

15. Gathwala, G. \& Sharma, S. Phototherapy induces oxidative stress in premature neonates. Indian J. Gastroenterol. 21, 153-154 (2002).

16. Tatli, M. M., Minnet, C., Kocyigit, A. \& Karadag, A. Phototherapy increases DNA damage in lymphocytes of hyperbilirubinemic neonates. Mutat. Res. 654, 93-95 (2008).

17. Yahia, S. et al. Influence of hyperbilirubinemia and phototherapy on markers of genotoxicity and apoptosis in full-term infants. Eur. J. Pediatr. 174, 459-464 (2015). 
18. Donneborg, M., Vandborg, P., Hansen, B., Rodrigo-Domingo, M. \& Ebbesen, F. Double versus single intensive phototherapy with LEDs in treatment of neonatal hyperbilirubinemia. J. Perinatol. 38, 154 (2018).

19. Kawai, K., Li, Y. \& Kasai, H. Accurate measurement of 8-OH-dG and 8-OH-Gua in mouse DNA, urine and serum: effects of X-ray irradiation. Genes Environ. 29, 107-114 (2007).

20. Horn, S., Barnard, S. \& Rothkamm, K. Gamma-H2AX-based dose estimation for whole and partial body radiation exposure. PLOS ONE 6, e25113 (2011).

21. Wu, L. L., Chiou, C., Chang, P. \& Wu, J. T. Urinary 8-OHdG: a marker of oxidative stress to DNA and a risk factor for cancer, atherosclerosis and diabetics. Clin. Chim. Acta 339, 1-9 (2004).

22. Kuo, L. J. \& Yang, L. X. Gamma-H2AX - a novel biomarker for DNA double-strand breaks. In Vivo 22, 305-309 (2008).

23. Barolet, D. Light-emitting diodes (LEDs) in dermatology. Semin. Cutan. Med. Surg. 27, 227-238 (2008)

24. Maisels, M., Kring, E. \& DeRidder, J. Randomized controlled trial of light-emitting diode phototherapy. J. Perinatol. 27, 565 (2007).

25. Kletkiewicz, H. et al. Effects of body temperature on post-anoxic oxidative stress from the perspective of postnatal physiological adaptive processes in rats. $J$. Physiol. Pharmacol. 67, 287-299 (2016).

26. Nuzum-Keim, A. \& Sontheimer, R. Ultraviolet light output of compact fluorescent lamps: comparison to conventional incandescent and halogen residential lighting sources. Lupus 18, 556-560 (2009).

27. Safari, S., Eshraghi Dehkordy, S., Kazemi, M., Dehghan, H. \& Mahaki, B. Ultraviolet radiation emissions and illuminance in different brands of compact fluorescent lamps. Int. J. Photoenergy 2015, 504674 (2015).

28. Moseley, H. \& Ferguson, J. The risk to normal and photosensitive individuals from exposure to light from compact fluorescent lamps. Photodermatol. Photoimmunol. Photomed. 27, 131-137 (2011).

29. Vreman, H. J. et al. Light-emitting diodes: a novel light source for phototherapy. Pediatr. Res. 44, 804 (1998).
30. Demirel, G., Uras, N. \& Celik, I. H. Comparison of total oxidant/antioxidant status in unconjugated hyperbilirubinemia of newborn before and after conventional and LED phototherapy: a prospective randomized controlled trial. Clin. Invest. Med. 33, 335-341 (2010).

31. Kale, Y., Aydemir, O. \& Celik, Ü. Effects of phototherapy using different light sources on oxidant and antioxidant status of neonates with jaundice. Early Hum. Dev. 89, 957-960 (2013).

32. Erel, O. A new automated colorimetric method for measuring total oxidant status. Clin. Biochem 38, 1103-1111 (2005).

33. Uchida, Y. et al. Phototherapy with blue and green mixed-light is as effective against unconjugated jaundice as blue light and reduces oxidative stress in the Gunn rat model. Early Hum. Dev. 91, 381-385 (2015).

34. Wagner, K. H. et al. Looking to the horizon: the role of bilirubin in the development and prevention of age-related chronic diseases. Clin. Sci. (Lond.) 129, 1 (2015).

35. Bulmer, A. C., Blanchfield, J. T., Toth, I., Fassett, R. G. \& Coombes, J. S. Improved resistance to serum oxidation in Gilbert's syndrome: a mechanism for cardiovascular protection. Atherosclerosis 199, 390-396 (2008).

36. Brito, M. A., Brites, D. \& Butterfield, D. A. A link between hyperbilirubinemia, oxidative stress and injury to neocortical synaptosomes. Brain Res. 1026, 33-43 (2004).

37. Kapitulnik, J. Bilirubin: an endogenous product of heme degradation with both cytotoxic and cytoprotective properties. Mol. Pharmacol. 66, 773-779 (2004).

38. Finkel, T. \& Holbrook, N. J. Oxidants, oxidative stress and the biology of ageing. Nature 408, 239 (2000).

39. Odell, G. B., Natzschka, J. C. \& Storey, G. Bilirubin nephropathy in the Gunn strain of rat. Am. J. Physiol. 212, 931-938 (1967).

40. Ebbesen, F., Hansen, T. W. \& Maisels, M. J. Update on phototherapy in jaundiced neonates. Curr. Pediatr. Rev. 13, 176-180 (2017). 